\title{
Incorporation of elevator evacuation from a specific floor - A numerical study of an office building
}

\author{
Johanna Hammarberg', Håkan Niva², Axel Mossberg ${ }^{3}$ \\ ${ }^{1}$ Briab - Brand \& Riskingenjörerna AB \\ Stockholm, Sweden \\ johanna.hammarberg@briab.se \\ ${ }^{2}$ Briab - Brand \& Riskingenjörerna AB \\ Malmö, Sweden \\ hakan.niva@briab.se \\ ${ }^{3}$ Brandskyddslaget AB \\ Stockholm, Sweden \\ axel.mossberg@brandskyddslaget.se
}

\begin{abstract}
This paper investigates evacuation from a specific floor plan to determine if elevators can replace one of the escape routes consisting of a staircase without lengthening the evacuation times. This study specifically studies evacuation from one single office floor with 360 and 540 occupants, designed as one fire compartment with an area of $2800 \mathrm{~m}^{2}$. To specify the occupants' willingness to use the elevator for evacuation from different floors, three different functions are used. These functions are modified to include more floors and are used to derive different distributions for the proportion using staircases and elevators, by simulations in Pathfinder. Two setups are studied. First, three staircases are used for evacuation. Secondly, one staircase is replaced with five evacuation elevators with capacity for eight occupants, and one elevator for sixteen occupants. For these setups, evacuation is studied from the $2^{\text {nd }}, 8^{\text {th }}, 16^{\text {th }}, 25^{\text {th }}$ and $50^{\text {th }}$ floor. In addition to the studied functions, distributions that minimize the evacuation time for each floor are derived from the simulations. These distributions give the shortest possible evacuation times. The distributions are then compared to evacuation simulations from the first setup, only utilizing the three staircases. The results show that for the elevators to fully replace a staircase, between $45-60 \%$ of the floor occupants need to use the elevators when the occupant count is 360 , and $43-50 \%$ when the number of occupants is 540. However, these values are dependent on floor number. Compared to the functions studied, the optimal percentage is significantly higher for lower floors, becoming closer to the functions as they increase with higher floor numbers. For each distribution on every floor, queuing time was also studied. Based on the results from the calculations, the study concludes that six evacuation elevators could replace one staircase on the studied office floor. However, this result relies on a certain percentage of the occupants using elevators for evacuation.
\end{abstract}

Keywords: elevator evacuation, evacuation modelling, pathfinder, evacuation, numerical study

\section{Introduction}

As urbanization increases, it is becoming more difficult to accommodate the need of living- and workspace within the cities. As a response to this, more focus is being put into constructing more highrise buildings. However, this development can lead to other issues, for example ensuring safe evacuation for the parts of the population that are not able to use the staircases by themselves. This could be solved by incorporating evacuation elevators in the building's evacuation strategy. However, to what extent the evacuation elevators will be used is still an unsolved issue. The main issue is the fact that people have been taught not to use elevators in case of a fire. When buildings are constructed in such a way that the evacuation strategy involves elevators, it may therefore be necessary to review people's approach and behavior in this matter. This issue is further complicated by the contradicting fact that people are used to evacuate the way that they entered the building, and in high-rise buildings the common way to enter would be with an elevator. The advantages that evacuation elevators offer makes it a relevant area to study further.

Since the terrorist attack on the World Trade Center, the focus on elevator evacuation strategies has increased within the fire research community [1]. Connected to this, awareness for robust and reliable 
elevator systems has been raised [2]. The solution with elevator evacuation is still relatively uncommon, as there are several challenges where more research is needed. Validation of the evacuation elevators' capacity and overcoming the general population's perception of elevator usage during evacuation are some of these challenges [3, 4].

Queuing is something that usually occurs when people evacuate. In case of evacuation from highrise buildings, queues can form when using stairs or elevators as an escape route. By queuing and prolonging the evacuation procedure, evacuees can be exposed to fire or smoke if the queuing occurs in the fire compartment where the fire started. Queuing can also lead to unrest and stressful situations if the evacuees are standing in line for too long.

Incorporating elevators in a building evacuation strategy is not only a matter of psychology and occupant flows. It is mainly a regulatory issue, as buildings always must follow the local regulations. Also, it is unlikely for elevator evacuation to be incorporated in buildings as a non-mandatory add-on to the fire safety as the cost of this installation might be quite substantial. In Sweden, there is a possibility to use evacuation elevators to replace one of the otherwise mandatory staircases that are built if equal evacuation safety can be proved. In order to prove this, the efficiency of elevator evacuation needs to be examined further.

Several previous studies have investigated the efficiency of elevator evacuation, but with the focus on total evacuation of a building, i.e. the evacuation of all occupants from every floor $[2,5,6]$. However, no studies have been found on how elevator evacuation affects the evacuation procedure from a single floor or fire compartment. In Sweden, the building regulations on fire safety do not include a provision to study the effects of a total evacuation but the evacuation of each fire compartment and floor needs to be secured separately. Because of this, this study investigates under what circumstances a number of evacuation elevators can replace a staircase for certain evacuation scenarios when studying a single office floor.

The study presented in this paper specifically studies the evacuation from one single office floor, designed as one fire compartment. One main factor of the study was the percentage of occupants in the building that would have to use the elevators depending on which floor that was evacuated. However, the effect of changing capacities and velocities for the elevators, and other more technical specifications are also briefly studied.

\section{Method}

The simulation software used in this study was Pathfinder, which is a commonly used evacuation tool developed by Thunderhead Engineering. In order to specify the occupants' willingness to use the elevator for evacuation from different floors, three different functions were used and incorporated in the simulations $[3,7,8]$. These functions are given as 1-3 below. These three functions were developed in different studies with the help of questionnaires to investigate the connection between which floor occupants were located on and how many of them were willing to evacuate with elevators. Simulations were done once per floor per function.
$P=0.84 * F+1.05$
$5<F \leq 24$
(1) Andersson and Jönsson [3]
$P=1.14 * F+5.3$
$5 \leq F \leq 60$
(2) Heyes [7]
$P=0.320 * \ln F-0.4403$
$5 \leq F \leq 55$
(3) Kinsey [8]

In these functions, $\mathrm{P}$ is the percentage of occupants using elevators for evacuation from the floor and $\mathrm{F}$ is the floor number being evacuated.

The functions were slightly modified to create three different evacuation scenarios for each studied floor. The functions were essentially extended to incorporate a larger range of floors. Function 1 was extended to be valid up to floor number 50 (from 24 in the original reference) and all three functions were assumed to have the same percentage of elevator usage on floor number two as for floor number five. The simulations used most of the program's standard settings [9], though occupant movement speed $(1.3 \mathrm{~m} / \mathrm{s})$ and person flow rate through openings $(1.1 \mathrm{p} / \mathrm{sm})$ were modified to be in line with the recommendations in the Swedish building regulation [10].

During the simulations, the evacuation of only one floor at a time was studied. This is the standard evacuation procedure for Swedish office buildings. The drawing in Fig. 1 was imported into Pathfinder where an estimated office environment with obstacles such as furniture, separating walls and pillars was 
drawn out. In the figure, the white area corresponds to the floor area where the occupants are placed at the beginning of the simulation. The floor area is about $2800 \mathrm{~m}^{2}$.

The studied office floor has several escape routes. As shown in Fig. 1, there are three staircases; left, centre and right. The central staircase has an adjacent elevator lobby with six elevators and three entrance points. Five of the six elevators are of the same size and have capacity for eight people, the sixth having twice this capacity. The staircases and the elevator lobby make up their own fire compartments and in order to reach the staircase in the middle occupants have to go through the elevator lobby. The floor area in the elevator lobby is approximately $60 \mathrm{~m}^{2}$ and the floor area in the two staircases on the sides are about $15 \mathrm{~m}^{2}$ each. The floor area of the central staircase is about $6 \mathrm{~m}^{2}$. Each staircase has a width of 1.2 meters, which is the required width of escape routes for fire compartments with more than 150 persons in the Swedish building code.

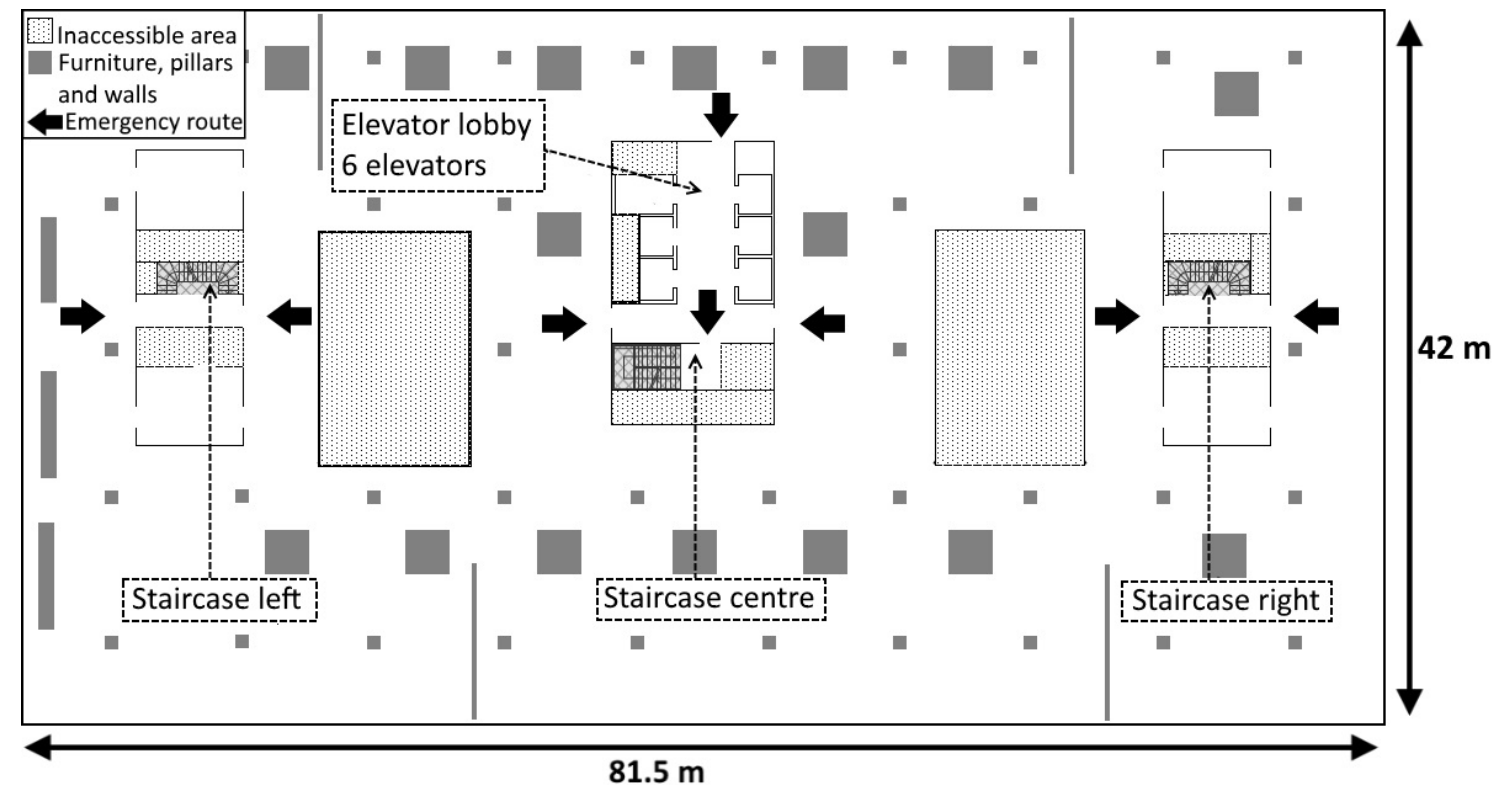

Fig. 1: Floor blueprint with items of significance.

The simulations studied evacuation from five different floors, i.e.: $2^{\text {nd }}, 8^{\text {th }}, 16^{\text {th }}, 25^{\text {th }}$ and the $50^{\text {th }}$ floor. This could also be regarded as the number of floors which the elevators would need to travel to get people to another safe floor, not necessarily the entrance floor. Two types of behaviors were used to define what emergency exit the occupants could use. One behavior only allowed for elevators to be used, and the other only allowed for stairs to be used. This made it possible to control what percentage of the occupants were going to use the elevators and the stairs.

To find out which values would be used for the elevators in Pathfinder, a few questions were sent to KONE via a contact form on the company's website. The values used were for "regular elevators" and are shown in Table 1. The delay between closing the elevator doors in the simulations until it begins to move was identified to be about 2.5 seconds by studying the elevators in the simulations. It was therefore not a setting chosen by the authors.

Table 1: Used elevator parameters.

\begin{tabular}{ll}
\hline Parameter & Used values \\
\hline Maximum occupants & 8 and 16 \\
Elevator velocities & $1.6,3,4$ and $10 \mathrm{~m} / \mathrm{s}$ (depending on \\
& floor number) \\
Elevator acceleration & $800 \mathrm{~mm} / \mathrm{s}^{2}$ \\
Time for doors to open and close & $5 \mathrm{~s}$ (total time for opening and closing) \\
Time between door closure and elevator movement & $2.5 \mathrm{~s}$ \\
\hline
\end{tabular}


In the first simulations, the evacuation of 360 occupants from the floor was studied. Additionally, simulations were performed with 540 people evacuating from the floor, which meant an increase of $50 \% .540$ was the allowed number of occupants on the floor according to the Swedish building code [11].

An assumption was made that as soon as occupants using the stairs reached the floor below, they were no longer part of the simulation as they had reached a safe zone. This meant that the simulation did not study the time it took to exit the building completely as this was deemed to give no further value to the study. This also means that factors like fatigue that can affect the evacuation from tall buildings were disregarded.

Two different evacuation setups were studied. In the first setup, the three staircases were used for evacuation. In the second setup, the centre staircase was replaced with six evacuation elevators. For these setups, evacuation was studied from the different floors mentioned above.

In addition to the different distributions mentioned above, "optimal" distributions for each floor were derived from the simulations. These "optimal" distributions were the ones giving the shortest possible evacuation times. These distributions were then compared to evacuation simulations from the first setup, only utilizing the three staircases. The reason that these distributions differ for different floors are explained by the fact that the elevator parameters differ according to Table 1 above and also that the travel distance for the elevator varies. The purpose with this comparison was to investigate if the six evacuation elevators could theoretically replace one staircase without lengthening the evacuation times.

The queuing time was also studied for the case with 360 occupants. The queuing time for elevator usage was the time from the first person who entered one elevator until the last person entered the elevator and the door closed. In occasional cases of evacuation from floor 25 and 50, 1-2 people did not fit with the last elevator round and had to wait for the last elevator to return, which could take up to 50 seconds. It was then assumed that those occupants actually took place in one of the last elevators as it was considered to be a more realistic situation. At most, the occupant density in the elevator lobby was as high as 3.2 persons per square meter in the simulations. This was calculated by Pathfinder and was not a factor that the study was heavily focused on, meaning it was not further investigated. The queuing time for staircase usage was the time from the moment a small queue was formed at the stairs, when people slowed down by those who were in front, until the last occupants could enter the first step of the staircase.

Queuing time for 540 occupants was not studied due to time constraints. Furthermore, the three studied distributions were not applied to the scenarios with 540 occupants, for similar reasons.

\section{Results}

The evacuation simulations show that in order to replace a staircase with evacuation elevators, the percentage using the elevators need to be increased in the lower floors, see Fig. 2, assuming that the distributions from the previous studies are correct. With the optimal distributions of evacuation elevator usage, the evacuation times from the floor are similar to when only staircases were used. 


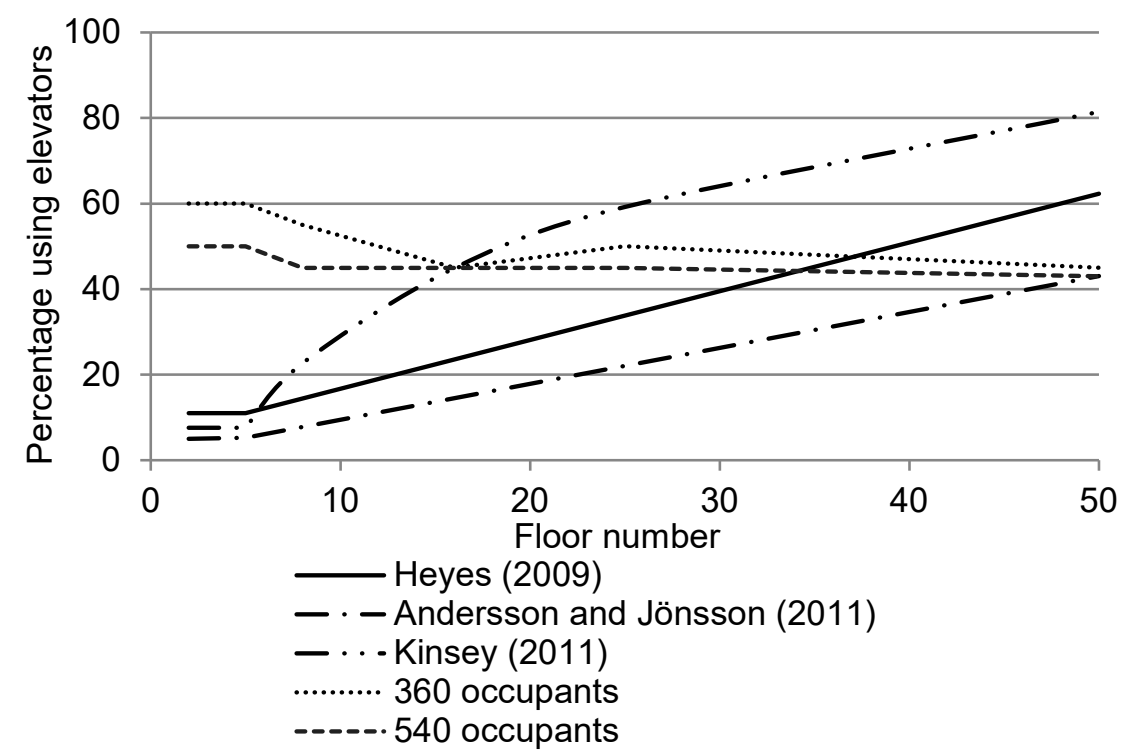

Fig. 2: Optimal distributions compared to correlations on elevator usage from different floors.

The simulations show that for 360 occupants, approximately $45-60 \%$ of the evacuees need to use the elevators for each floor to acquire similar evacuation times as if only using the staircases. When the number of occupants increases to 540 , between $43-50 \%$ of the evacuees need to use the elevators in order to get equivalent times as the staircase solution. This is also indicated by the results in Fig. 2, which shows that the scenarios with 540 occupants evacuating from the floor gives a slightly lower optimal percentage of evacuees using the elevator.

Results from the simulations with 360 occupants show that queuing times for the evacuation elevators increase significantly for some of the functions when the floor that was evacuated was higher up in the building. This is natural, as the elevator usage also increases with floor number in the correlations. However, the significance of the difference with these solutions have previously not been quantified. These results are shown in Fig. 3. Note that the evacuees did not change evacuation route, regardless of waiting/queuing times.

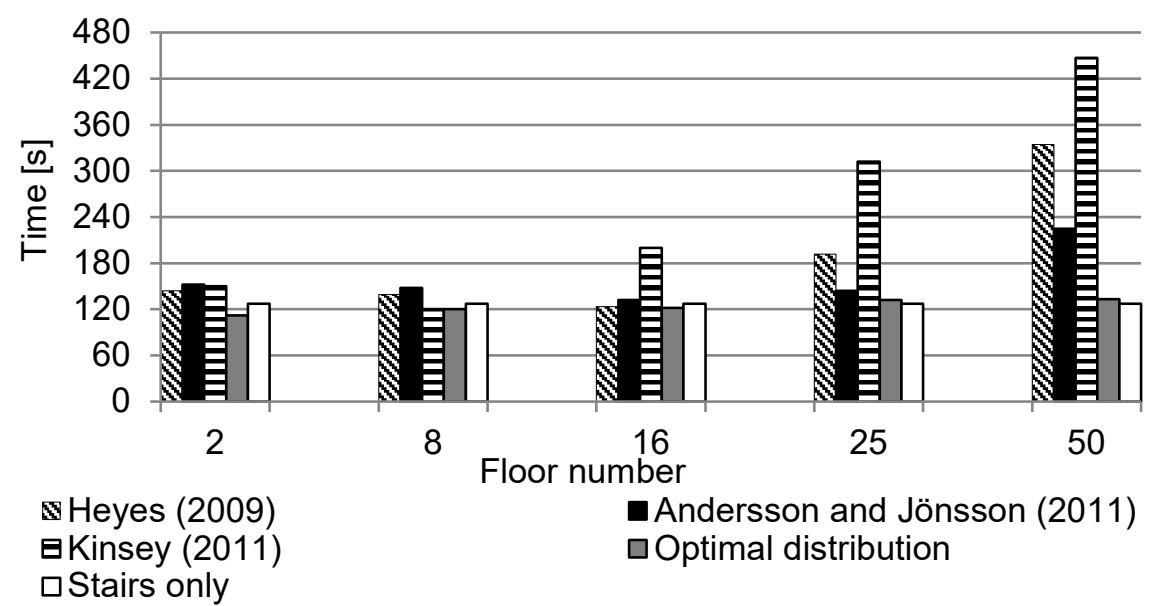

Fig. 3: Queueing times for the different correlations and floors studied for 360 occupants. Here, the maximum queueing time during the simulation is displayed.

The results also show that as the studied floor number increases, the required time for all occupants to reach another fire compartment generally decreases, with one major exception, as shown in Fig. 4. In general, this is because of the increasing elevator usage and the available occupant capacity of the elevator lobby. When elevator usage increases, it takes less time for the occupants to reach a new fire compartment since some of those who previously were queuing to the staircases now can enter the elevator lobby instead. The exception to this is with function 3 (Kinsey, [8]) on floor 50, which has the highest elevator usage of around $80 \%$. Here, the capacity of the elevator lobby is not enough to accommodate for the high number of occupants using the elevators, which leads to queuing to the lobby 
while the staircases are free of queues instead. In all simulations it was concluded that if the optimal distributions where applied, the results were similar to when only staircases were used for evacuation.

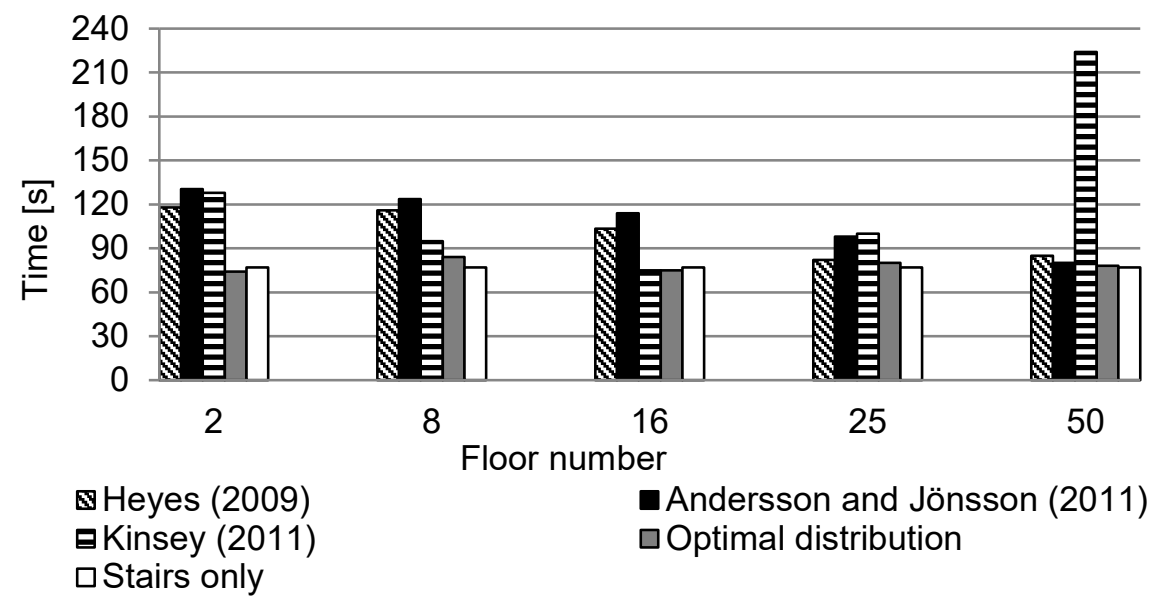

Fig. 4: Time until all 360 occupants had reached the next fire compartment.

When the number of occupants was increased to 540 and simulations were done to study how well the optimal distributions for 360 occupants could be directly applied to the increased number of occupants, the results show that the distributions are not directly applicable for different number of occupants, as seen in Fig. 5. The difference in the time it took for the occupants using elevators to evacuate compared to the ones using staircases is significant. Therefore, new distributions were developed, which can be seen in Fig. 2. This indicates that, in this case, the number of occupants impacts the optimal distribution of elevator usage.

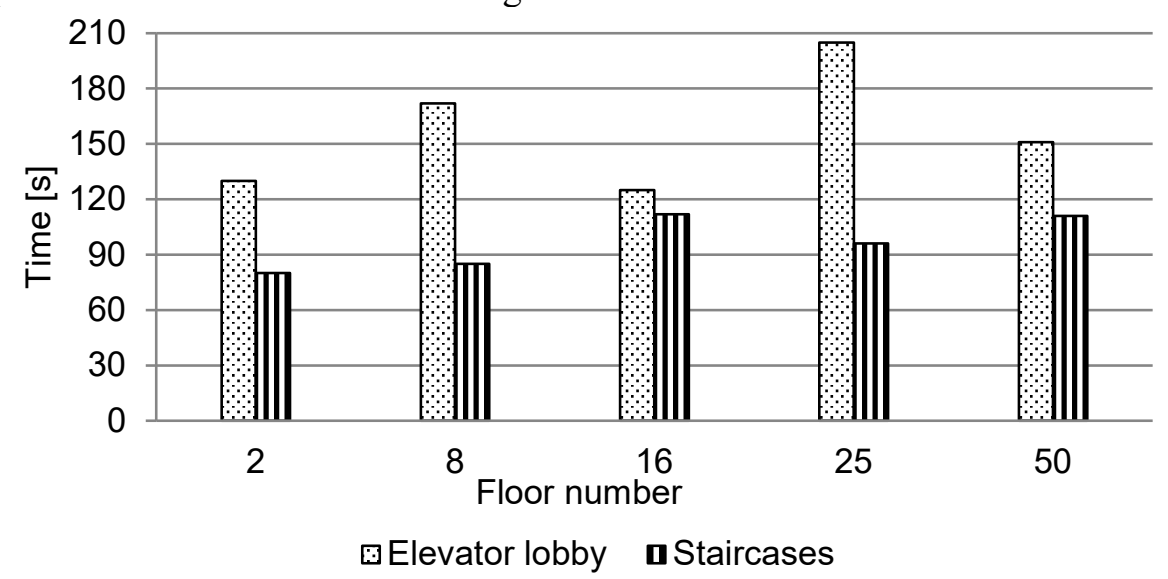

Fig. 5: Optimal distributions for 360 occupants applied to 540 occupants, showing the difference in evacuation time for elevator and staircase users.

With the optimal distributions for 540 occupants developed the same way as before, Fig. 6 shows a comparison of the evacuation scenarios with elevators and stairs to the scenarios when only the staircases were used. With the exception of evacuation from the $2^{\text {nd }}$ floor, the results show similar required time for the occupants to reach the next fire compartment. With the optimal distributions, the required time for evacuating the $2^{\text {nd }}$ floor was around 30 seconds shorter than when only staircases were used. 


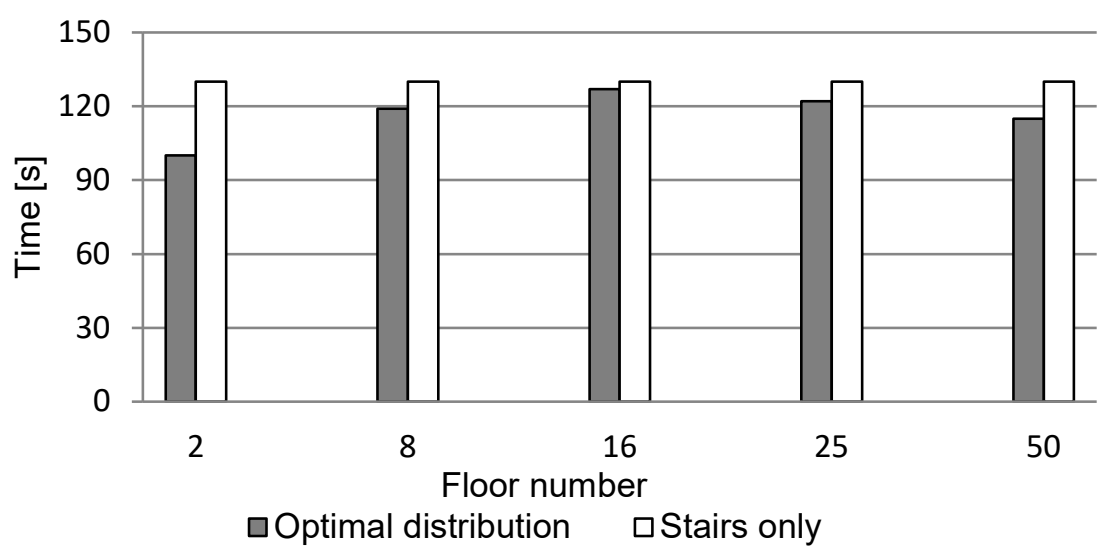

Fig. 6: Comparing the time until all 540 occupants had reached the next fire compartment, using optimal distributions and only the staircases.

\section{Discussion}

The optimal distributions for the most efficient evacuation proved to be more constant than the distributions of occupants' willingness to use elevators from previous studies, which increased with higher floors. There seem to be breakpoints where a higher proportion than expected from the previous studies need to evacuate by elevator on lower floors in order for the elevators to be effective. Similarly, with higher floors, a higher number of staircase usage would be needed in order for the elevator solution to be more effective.

Naturally, when the number of occupants increased from 360 to 540, time to another fire compartment increased too. When the simulations were made for 540 occupants the same distributions as for the scenarios with 360 occupants were used. This showed that time to another fire compartment for the scenarios with elevators increased and did not give the same results as for 360 occupants. Occupant times to reach another fire compartment for the stairs were similar at the lower floors, but at higher floor levels this time increased. In order for the scenarios with evacuation elevators and the scenarios with only stairs to be completed at the same time with 540 occupants, a smaller proportion should use the elevators than indicated in the distributions for the 360 occupants. In summary, this result implies that with a change of occupant number, new optimal distributions need to be derived in order to find the optimal evacuation procedure.

When the optimal distributions for 360 and 540 occupants were compared with the scenarios when only the stairs were used, the evacuation times were similar. This means that the elevators used for the simulations can replace the staircase for this specific floor.

As Fig. 6 shows, the time for the optimal distributions for 540 occupants are always slightly lower than the time to use only stairs. This could indicate that if increasing the occupant number, the evacuation can be more effective if one staircase is replaced with six elevators. However, this needs more studies to be concluded.

\section{Conclusions}

Based on the results from the performed calculations, the study concludes that one staircase on the studied office floor could be replaced with six evacuation elevators. However, this result relies on $45-60 \%$ or $43-50 \%$ of the occupants using elevators for evacuation, for 360 and 540 occupants respectively. These distributions represent optimal distributions between the elevator and stair users in the studied evacuation situations.

When compared to functions derived in previous studies on human behavior connected to elevator evacuation, these results pose certain challenges, i.e., on lower floors, the number of occupants using the evacuation elevators needs to be increased in order for the solutions to be equivalent. This means that on these floors, the usage of the elevators needs to be promoted. However, at higher floor numbers, the problem is the opposite, as too many occupants are willing to use the evacuation elevators. Here, the stairs may need to be promoted in order for the evacuation to be as effective as possible. The results of this study highlight the importance to further study how to incentivize occupants to use both elevators and stairs in high-rise buildings. 
This study relates to the layout of the certain office building used. Therefore, the results cannot be directly translated to other buildings. However, the conclusions mentioned above can be assumed to be general indications to expect in any building utilizing elevators for evacuation. Hence, the study highlights the importance of dealing with these problems in the fire safety design.

\section{References}

[1] D. Nilsson and A. Jönsson, "Design Evacuation Systems for Elevator Evacuation in High-rise Buildings," Journal of Disaster Research, vol. 6, pp. 600-609, 2011.

[2] E. Ronchi and D. Nilsson, "Modelling total evacuation strategies for high-rise buildings," Building Simulation, vol. 7, no. 1, pp. 73-87, 2014.

[3] J. Andersson and A. Jönsson, "Evacuation in High-rise Buildings - An analysis of risk perception," Lund University, Lund, 2011.

[4] M. Arnqvist and J. Olsson, "Emergency elevators as a means of egress - An analasys of the possibility to use elevators when designing emergency egress systems," Lund University, Lund, 2010.

[5] J. H. Klote, "A Method for Calculation of Elevator Evacuation Time," Journal of Fire Protection Engineering, vol. 5, no. 3, pp. 83-95, 1993.

[6] E. Kuligowski, "Elevators for Occupant Evacuation and Fire Department Acces," Kuala Lumpur, 2003.

[7] E. Heyes, Human Behavior Considerations in the Use of Lifts for Evacuation from High Rise Commercial Buildings, Canterbury: University of Canterbury, 2009.

[8] M. J. Kinsey, "Vertical transport evacuation modelling," University of Greenwich, Greenwich, 2011.

[9] Thunderhead Engineering, "Pathfinder User Manual," Thunderhead engineering, New York, 2014.

[10] Boverket, "BBRAD 3," Boverket, Karlskrona, 2013.

[11] Boverket, "BFS 2014:3," Boverket, Karlskrona, 2014. 